\title{
Performance Evaluation of a MIMO based Underwater Communication System under Fading Conditions
}

\author{
Boddu Pranitha \\ Department of Electronics and Communication Engineering \\ National Institute of Technology \\ Warangal, Telangana, India \\ pranitha.boddu@nitw.ac.in
}

\author{
L. Anjaneyulu \\ Department of Electronics and Communication Engineering \\ National Institute of Technology \\ Warangal, Telangana, India \\ anjan@nitw.ac.in
}

\begin{abstract}
Due to the intense importance of underwater applications in military and commercial purposes, Underwater Acoustic Communication (UWAC) is an attracting research area. Very high propagation delay, path loss, and low bandwidth are the factors that challenged the acoustic waves in an underwater environment. Multi-Input Multi-Output (MIMO) techniques are currently considered in UWAC to surpass the bandwidth limitation of the undersea channel. In this paper, the accomplishment of a MIMO in UWAC System highlighting both Line of Sight (LOS), i.e. the Rician fading and Non-Line of Sight (NLOS), i.e. the Rayleigh fading signal propagation, is assessed. Spatial Modulation technique is used, which helps in increasing the data rate in UWAC. It controls the spatial distribution of the energy caused by a signal in such a way that the single ocean channel sustains multiple parallel communication channels. The utilization of Zero Forcing (ZF) equalizer, which estimates the transmitted data proves the success of removing Inter Symbol Interference (ISI). Matlab simulations are done for the UWAC system for values of LOS/NLOS. Because of various scattering effects in NLOS propagation, the error rate is considerably high when compared to that of the LOS propagation. Bit Error Rate (BER) values for the corresponding Signal to Noise Ratio (SNR) are calculated.
\end{abstract}

Keywords-Underwater Acoustic Communications (UWAC); Multiple Input Multiple Outputs (MIMO); Rayleigh fading; NonLine of Sight (N-LOS); Rician fading; Line of Sight (LOS)

\section{INTRODUCTION}

Nowadays, Underwater Acoustic Communications (UWAC) is an open research, which includes the present day expansion of human activities in undersea environments, i.e. environmental monitoring and offshore oil field exploration. Robotics in underwater environment is also becoming a major aspect of research in UWAC in order to increase precision [1]. Today, underwater remote sensing systems such as SONARs are used extensively for detecting and tracking different targets using sound signals [2]. Recently, significant attention has been given to the Underwater Wireless Sensor Networks (UWSNs) because of their applications in commercial, scientific, environmental and military purposes [3-4]. The performance and reliability of the system have been improved due to the continuing research. Because of underwater high attenuation and absorption effect, electromagnetic waves can travel only short distances [5]. Some investigations have been made about utilizing optical signals for underwater applications. However, it was found that they can only pass through a limited range in a very clean water environment, making them useless for underwater long-distance transmissions [6]. Thus, acoustic signals are found to be the only physical feasible way that works in an underwater environment reducing bandwidth limitations [7]. High propagation delay, heavy energy consumption, large path loss, and low bandwidth have posed challenges for applying acoustic waves in underwater environment [8]. Three major factors that characterize acoustic wave propagation are attenuation, multipath propagation that varies along with time, and low sound speed. Attenuation increases with the frequency of the signal [9]. Fading, usually called small scale fading, is caused by multipath signals. The signal may be constructive or destructive [10]. Generally, these factors limit system performance. Three factors that characterize the channel in UWAC are long multipath delay, high Doppler spread, and time-varying Doppler shift. This multipath delay is the main cause of Inter-Symbol Interference (ISI). Doppler spread, and Doppler shift are caused due to relative speed that is comparable with the speed of sound [11]. Severely low bandwidth, large area multi-path spread, Doppler shift, and background noise make the underwater environment much more complicated than a simple wireless channel. Hence, the design and implementation of high data rate UWAC are a major challenge.

Influence of interference in Wireless Communication has been studied in fading environment using outage probability calculation [12]. In low frequency (i.e. $<1 \mathrm{kHz}$ ) sound propagation, the fading components, Rayleigh and Rician are related with saturated and partially saturated schemes respectively in which the multipath is completely or partially random. The propagation of acoustic waves requires a medium to transmit data. This medium can be either shallow or deep water. Irrespective of the type of the medium, propagation loss and fading exists. Propagation loss, also known as path loss is the value of the power of the receiver at the receiving end. 
Distance in the propagation (considering LOS and NLOS), water density, strong currents, waves, etc. are factors that impact path loss. Because of the presence of living and nonliving objects underwater, a portion of the acoustic signal gets either reflected or diffracted and scattered. This is referred to as slow fading in UWAC, while fast fading is caused due to multipath propagation loss. Considering all these issues, the main focus of our research is to characterize all the mentioned properties in the UWAC channel. Estimating the transmitted signal in UWAC is to be studied as well [13].

Multiple Input Multiple Output (MIMO) is the advanced and sophisticated technology which is adapted from land wireless communication to underwater communication because of the increasing demand for high data rates. In this paper, this technique is used which helps in reducing the interference of UWAC systems. Also, spectral efficiency in multiple transmitters can be increased [14]. MIMO techniques are applied to UWAC with spatial modulations [15]. MIMO approaches have taken advantage of available channel equalization algorithms that are used for single carrier transmissions [16]. The bandwidth of the transmitting and receiving means of MIMO occupy the same frequency band, without the use of any further receiver transmitting power. This can increase the capacity of the channel and lessen the Bit Error Rate (BER) of the system [17]. Whenever the transfer functions between different transmitting and receiving antennas are identically and independently distributed (i.i.d.) and their significances are explained by the Rayleigh distribution, then an ideal situation arises for a MIMO system. This distribution describes the conditions for NLOS signal propagation. However, it can be noted that UWAC scenarios deviate from the given ideal conditions as they give signal propagation details of LOS and NLOS which are expressed as Rician distribution and Rayleigh distribution respectively. To recover the data that is transmitted, Zero Forcing (ZF) equalization technique is used. An equalizer is a digital filter used to mitigate the effects of ISI introduced by a time dispersive channel. MIMO linear equalizer is used to attain lowcomplexity equalization. It reduces the error propagation. $\mathrm{ZF}$ equalizer is a linear equalization algorithm used in communications. It generally affixes the inverse of the frequency response of the channel matrix. This technique is used when ISI is high compared to the noise in the system. This technique is generally ideal in the case of noiseless channels. A ZF equalizer controls the ISI and ICI introduced by the transmission channel. This equalizer does not take enhancement of the noise into consideration [18].

In our previous work [19], a comparison for ZF equalizer and LMS equalization has been made in UWAC for a $2 \times 2$ MIMO in which ZF equalization outperforms LMS equalization technique. In this paper, the $\mathrm{ZF}$ equalization technique at the receiver will be compared for Rayleigh and Rician fading UWAC MIMO channels. Rician fading includes the LOS while the Rayleigh fading has the N-LOS component considering multipath of UWAC in it. All the losses included in UWAC channels such as spherical loss and absorption loss were considered. The Doppler effect and the relative motion that exists between the transmitter and receiver were also considered. LOS and N-LOS links consider that the wave reflections are not significant, i.e. the transmitter and receiver are neither near to water surface nor to the seabed. Matlab simulations have been carried out for all these conditions, and the results were compared.

\section{SYSTEM MODELING}

To reduce bandwidth limitation to the most extent of the undersea channel, MIMO is considered in UWAC. Multiple antennas are used by MIMO technology to acquire various gains namely multiplexing and beam forming. Nonetheless, the complexity and cost of the receiver are increased because of these gains. Spatial Modulation (SM) is one good approach to overcome the problems mentioned above. In SM, the information bits are mapped into two different constellations: a signal constellation and a spatial constellation. Signal constellation is based on the modulation scheme, while the spatial constellation is used to encode the index of the transmitter. At any point in time, one of the transmitters remains active, while the other transmitter radiates zero power. Thus, SM avoids the Inter Channel Interference (IChI) at the receiver and reduces the high requirement of synchronization among transmitters. Moreover, unlike the regular MIMO system, RF chains are not required in $\mathrm{SM}$ at the transmitter [20]. In this part, we start by giving an introduction to SMMIMO concept in UWAC. This modulation scheme is applied to the transmitting or receiving antenna to send extra information, and so SM is used to find the index of the active antennas at any time instance. Therefore, the information bits that are to be transmitted are branched into two different parts. In the first part, they are graphed to a symbol from the signaling constellation. Here the type of modulation used finds the number of bits per symbol. From the set of receivers available for transmission or reception, the second part of the information bits regulate the index of the receiver that is selected from a set of receivers that are available for transmitting or receiving the data. Receiver selection in any general MIMO system depends on the state of the channel and the signal strength of the receiver. In SM-MIMO it depends on the incoming user data stream [21].

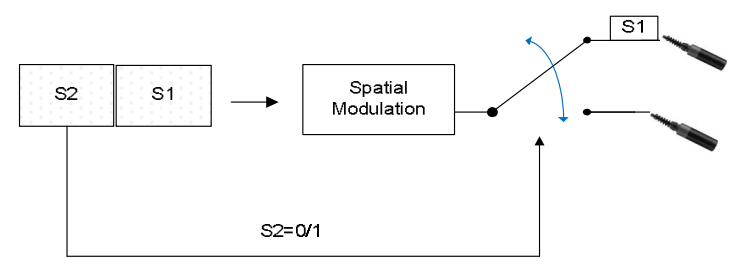

Fig. 1. Spatial modulation

The number of transmitters and receivers is $N_{t}$ and $N_{r}$ respectively. $M$ is the number of entities of the signal constellation. Here we are considering a 4-QAM modulation because of its bandwidth efficient quality. By finding the index of the active transmitter in SM-MIMO, one symbol S1 is transmitted directly, and the other symbol S2 indirectly in each channel use. For subjective $N_{t}$ and $M$, the rate of SM is:

$$
R_{S M}=\log _{2} M+\log _{2} N_{t}
$$


Hydrophones are used as transmitters as shown in Figure 1. Higher throughput, simpler receiver and transmitter design, and lower transmit power supply are the main advantages of SMMIMO. But the antenna has to be switched very quickly in this case. Here, a basic 4 QAM modulation is used. The received data for the $2 \times 2$ MIMO is given by:

$$
\left[\begin{array}{l}
y_{1} \\
y_{2}
\end{array}\right]=\left[\begin{array}{ll}
h_{11} & h_{12} \\
h_{21} & h_{22}
\end{array}\right]\left[\begin{array}{l}
x_{1} \\
x_{2}
\end{array}\right]+\left[\begin{array}{l}
n_{1} \\
n_{2}
\end{array}\right]
$$

where $y_{1}, y_{2}$ are the received data from the two receivers, $h_{11}$, $h_{12}, h_{21}$, and $h_{22}$ are the channel coefficients explained below, $x_{l}$ and $x_{2}$ are the transmitted data and $n_{1}$, and $n_{2}$ represent the UWAC noise. To detect the data at the receiver, a ZF equalizer is used. Detecting the information from the original noisy data being received is known as detection. MIMO detectors/receivers include a merge of many algorithms carrying out many tasks like estimation, detection, equalization, decoding, etc. [22]. In this paper, the ZF equalizer, which is based on channel matrix estimation, is put forward. The original system is divided into several subsystems allowing data estimation with practicable complexity. The inverse of the channel matrix is calculated and multiplied with the received data to obtain the transmitted data. The estimated/detected data is given by:

$$
\begin{aligned}
& \hat{x}=H^{-1} Y \\
& \hat{x}=H^{-1}(H x+n) \\
& \hat{x}=x+H^{-1} n
\end{aligned}
$$

$\mathrm{ZF}$ technique is less complex. The number of receivers should be equal to or greater than the number of transmit antennas in the case of ZF. However, it comes at the cost of increasing the power of noise that leads to power degradation [23]. When the Doppler frequency is small, the received signal quality will be better than when it is large. Consequently, it will influence the received signal quality. Interference between terminals can be suppressed further by using the ZF equalizer.

\section{CHANNEL MODELING}

The UWAC channel is highly complex because of its nonhomogenous nature. Water densities, temperature gradients, and non-homogeneities caused by the dropping particles of either solid or gaseous matter are the main reasons for most imperfections. The constant flow of water and the channel reflections that are caused at the sea surface and bottom increase the complexity further. This effect of the underwater channel is that it has strong multi-path propagation and the Doppler spread. However, salinity, temperature, and pressure help in finding the propagation velocity, which makes the transmission medium highly refractive [24]. The received signal combines a large number of multipath arrivals, especially scatterers formed by ocean surface and bottom. Such fading is known as a Rayleigh fading channel. Rayleigh fading channel is a shallow water channel commonly used, but when discrete arrivals are seen in the channel response, it imposes a challenge to make assumptions [25]. Point-to-point communications in UWAC are a major research area that has to be studied. This is known as LOS communications to underwater multicarrier modulation networks. Because of the presence of multipath scattering in the UWAC channel, the application of MIMO is promising in this case. Large fractional bandwidth is one of the main difficulties associated with UWAC. The acoustic multipath propagation in Rayleigh fading model follows an exponential distribution at the received power, while in a LOS propagation path, the Rician fading model is adapted [12]. When the Rayleigh fading model is used, the scatterers that are explained in Figure 2 are considered.

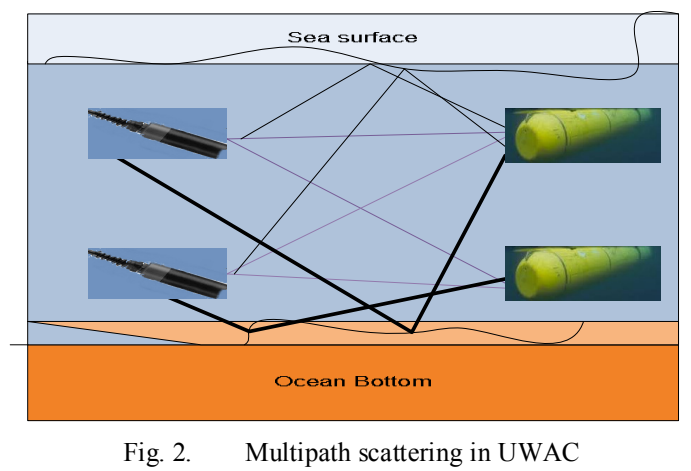

In this type of channel, no straight path exists between the transmitter and the receiver, but at the receiver, the sums of all the reflected and scattered waves are combined. Scattering may be mainly caused due to reflections from the sea surface and the ocean bottom. At the receiver signals are received in four different ways. They are the direct LOS direction between the transmitter and the receiver, the reflected signal from the bottom of the ocean surface, and the reflected signal from both surface and bottom. However, each receiver receives multiple reflected and direct signals from each transmitter. Figure 2 depicts the scattering scenario in UWAC. Rough sea surface or the seafloor causes attenuation of the acoustic field in an ocean waveguide. Here, attenuation increases with increasing frequency. Ambient noise is considered in the Rayleigh fading UWAC channel due to turbulence, shipping, ocean waves, and thermal noise [26]. The power spectral density (PSD) of these noise components as a function of frequency in $\mathrm{kHz}$ is expressed as:

$$
\begin{gathered}
\log N_{t}(f)=1.7-3 \log f(6) \\
\log N_{s}(f)=4+2\left(s-\frac{1}{2}\right)+2.6 \log f-6 \log (f+0.03) \\
\log N_{w}(f)=5+0.75 w^{1 / 2}+2 \log f-4 \log (f+0.4) \\
\log N_{t h}(f)=-1.5+2 \log f
\end{gathered}
$$

The shipping coefficient $s$ varies from 0 to 1 , for low and high action respectively. $w$ is the wind speed $(\mathrm{m} / \mathrm{s})$ [27]. The final PSD of the background noise is given by:

$$
N(f)=N_{t}(f)+N_{s}(f)+N_{w}(f)+N_{t h}(f)
$$

where $N_{t}(f)$ is the noise due to turbulence, $N_{s}(f)$ the noise due to the shipping activity, $N_{w}(f)$ is caused due to waves and $N_{t h}(f)$ is the thermal noise.

In a Rician fading channel, the LOS component is mainly due to ocean currents, pressure and temperature gradients, and 
salinity variations. The channel coefficients of UWAC in LOS are calculated as:

$$
h=\sqrt{\frac{c_{R}}{1+c_{R}}} A_{s}(d) A_{a}(d) e^{j\left(2 \pi f_{0} t+\theta_{0}\right)}
$$

where $c_{R}$ is the rician factor, $A_{s}(d)$ is the propagation loss coefficient due to spherical spreading, and $A_{a}(d)$ is the absorption loss. $f_{0}$ gives the Doppler frequency and $\theta_{0}$ represents the phase shift of this LOS component [28]. While evaluating the channel coefficients, all the factors involved in effecting the performance of the system are considered. Due to the motion between the source, the receiver and the inhomogeneous ocean medium, sound waves are affected by Doppler shift which results in frequency spread in the received signal [29].The Doppler frequency $f_{0}$ is expressed as:

$$
f_{0}=f_{\max } \cos \left(\alpha_{0}-\alpha_{v}^{R}\right)
$$

where $f_{\max }$ is the maximum Doppler frequency given by:

$$
f_{\text {max }}=v_{R} f_{c} / c_{s}
$$

where $v_{R}$ is the receiver's speed, $f_{c}$ gives the carrier frequency, and $c_{S}$ denotes the sounds speed, which is generally considered as $1500 \mathrm{~m} / \mathrm{s}$. The factors $\alpha_{0}$ and $\alpha_{v}{ }^{R}$ are considered when there is an angle of arrival between the transmitter and the receiver. Different water properties such as temperature, salinity and pressure impact the speed of sound in water. The speed of sound in water near the surface is approximately 4 times faster than the speed in the air.

The propagating signal phase shift can be calculated as:

$$
\theta_{i j}=\frac{d_{i j}}{2 \pi} * \lambda
$$

where $i$ is the $i^{\text {th }}$ transmitter and $j$ is the $j^{\text {th }}$ receiver. The wavelength $\lambda$ is given by

$$
\lambda=1500 / f_{c}
$$

If the signal gets weakened because of the geometrical spreading of a wave propagating away from the source, then the created loss is known as spreading loss. Different kinds of non-uniformities are present in the sea and within its boundaries. These inhomogeneities reradiate a portion of the acoustic energy that is incident upon them which is known as scattering. Due to the presence of geometrical spreading and absorption spreading, as an acoustic wave propagates through the medium, its intensity is reduced. However, there is a drastic change in the attenuation of acoustic waves in water when compared to the RF waves in water. Also there is a heavy loss of energy in water due to absorption. The loss that is caused by geometrical spreading can be either spherical or cylindrical. The loss due to spherical spreading is calculated by:

$$
A_{s}(d)=\frac{1}{d}
$$

where $d$ is the distance between the transmitter and the receiver.

During sound wave propagation, energy can be converted to many other modes, and the medium absorbs it. The propagating physical wave controls the absorptive energy loss.
The absorption loss according to the Schulkin-Marsh model is calculated as:

$$
A_{a}(d)=10^{\frac{-d \beta}{20000}}
$$

The parameter $\beta$ is given by:

$$
\beta=8.68 * 10^{3}\left(\frac{S_{a} f_{T} f_{c}^{2} A}{f_{T}^{2}+f_{c}^{2}}+\frac{B f_{c}^{2}}{f_{T}}\right) *\left(1-6.54 * 10^{-4} P\right)
$$

where $A$ and $B$ are constants, $S_{a}$ stands for salinity, $f_{c}$ for carrier frequency and $f_{T}$ is the relaxation frequency which is given by:

$$
f_{T}=21.9 * 10^{6-\left(\frac{1520}{T+273}\right)}
$$

Here $T$ is the hydrostatic temperature given in ${ }^{\circ} \mathrm{C}, P$ denotes the hydrostatic pressure which depends on water depth $h_{t}$, which is given by:

$$
P=1.01\left(1+0.1 h_{t}\right)
$$

In Figure 3, two transmitters are placed apart at a distance $L_{1}$, while $d_{11}$ is the distance between the transmitter and the receiver, and $L_{2}$ is the distance between the two receivers. It is assumed that the transmitters and receivers are in parallel planes and $L_{1}>L_{2}$. For calculating the channel coefficients $\left[\begin{array}{ll}h_{11} & h_{12} \\ h_{21} & h_{22}\end{array}\right]$ using (2), we consider distances $d_{11}, d_{12}, d_{21}$, and $d_{22}$ respectively. Assuming that the distances $d_{11}, L_{1}$ and $L_{2}$ are fixed, the remaining distances could be calculated. The signal phase shift $\theta_{i j}$ can also be determined for various distances.

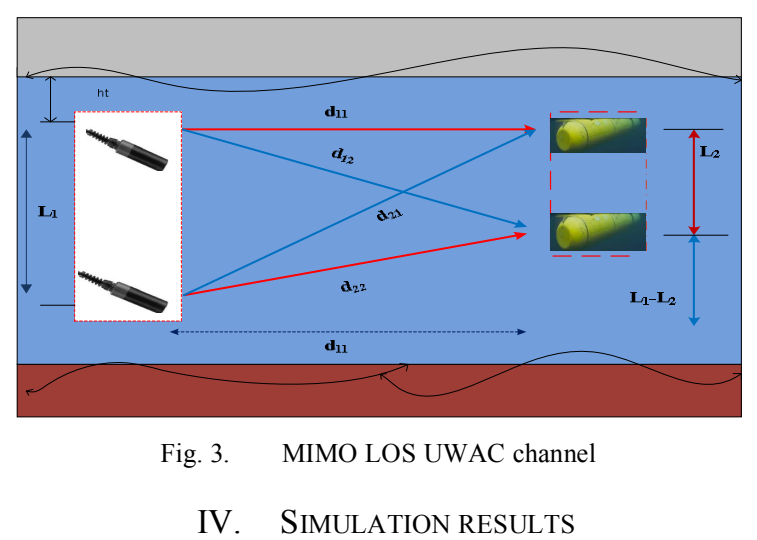

Simulations in Matlab have been carried out for the channel model shown in Figure 3. 4-QAM spatial modulation is given at the transmitter which is a cost and complexity efficient, while at the receiver $\mathrm{ZF}$ equalization is utilized to detect the transmitted data. For NLOS, i.e. the Rayleigh fading considers the multipath fading involved in UWAC. The ambient noise which involves noise caused due to shipping, waves, turbulence, and thermal noise is also considered. While in LOS, Rician fading factors such as absorption loss, spherical loss, temperature, pressure, salinity, Doppler frequency, etc. are considered as shown in Table I. Simulations were carried out for both LOS and N-LOS UWAC, i.e. for both Rician and Rayleigh fading channels. Figure 4 depicts that Rician fading outperforms the Rayleigh fading channel. The SNR to BER values are tabulated in Table II. 
TABLE I. SIMULATION PARAMETERS

\begin{tabular}{|c|c|c|}
\hline Symbol & Parameter & Value \\
\hline $\boldsymbol{N}_{\boldsymbol{t}}$ & Number of transmitters & 2 \\
\hline $\boldsymbol{N}_{\boldsymbol{r}}$ & Number of receivers & 2 \\
\hline $\boldsymbol{N}$ & Number of transmitted bits & 10000 \\
\hline $\boldsymbol{m}$ & QAM order & 4 \\
\hline $\boldsymbol{c}_{\boldsymbol{R}}$ & Rice factor & 0.56 \\
\hline $\boldsymbol{d}_{\boldsymbol{1}}$ & Distance & $1 \mathrm{~m}-100 \mathrm{~m}$ \\
\hline $\boldsymbol{f}_{\boldsymbol{c}}$ & Carrier frequency & $10 \mathrm{kHz}$ \\
\hline $\boldsymbol{T}$ & Temperature & $10^{\circ} \mathrm{C}$ \\
\hline $\boldsymbol{S} \boldsymbol{a}$ & Salinity & $35 \mathrm{PSU}$ \\
\hline $\boldsymbol{P}$ & Pressure & $11 \mathrm{~kg} / \mathrm{cm}$ \\
\hline $\boldsymbol{c}_{\boldsymbol{s}}$ & Speed of sound & $1500 \mathrm{~m} / \mathrm{s}$ \\
\hline $\boldsymbol{L}_{\boldsymbol{l}}$ & Distance between transmitters & $5 \mathrm{~m}$ \\
\hline $\boldsymbol{L}_{\boldsymbol{2}}$ & Distance between receivers & $2 \mathrm{~m}$ \\
\hline $\boldsymbol{v}_{\boldsymbol{R}}$ & Speed of the receiver & $9 \mathrm{~m} / \mathrm{s}$ \\
\hline $\boldsymbol{h}_{\boldsymbol{t}}$ & Shallow water depth & $90 \mathrm{~m}$ \\
\hline
\end{tabular}

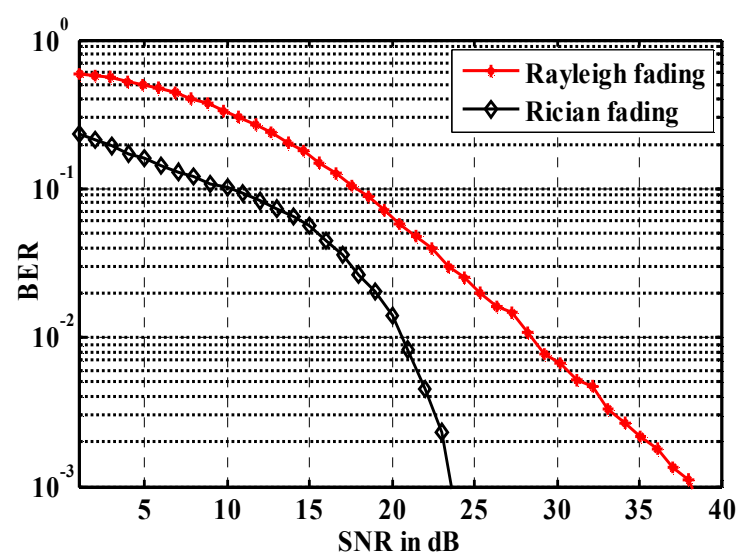

Fig. 4. SNR vs. BER of Rayleigh and Rician fading UWAC

TABLE II. BER VALUES

\begin{tabular}{|c|c|c|}
\hline \multirow{2}{*}{ SNR in dB } & \multicolumn{2}{|c|}{ BER } \\
\cline { 2 - 3 } & Rician Distribution & Rayleigh distribution \\
\hline 1 & 0.830409 & 0.94646 \\
\hline 5 & 0.782244 & 0.915555 \\
\hline 10 & 0.731129 & 0.870501 \\
\hline 15 & 0.658818 & 0.789072 \\
\hline 20 & 0.474383 & 0.689473 \\
\hline 25 & 0.020281 & 0.571698 \\
\hline 30 & 0.00 & 0.447859 \\
\hline 35 & 0.00 & 0.324006 \\
\hline 39 & 0.00 & 0.191 \\
\hline
\end{tabular}

From the above-tabulated values, we can observe that the error rate for Rayleigh distribution is very high when compared to Rician distribution. This is because the scattering effects are very less in the LOS component than in the N-LOS and also the presence of multipath fading affects the performance of the system. From Table II, we notice that the BER values of Rician distribution are zero for SNR values at $30 \mathrm{~dB}$. However, the BER value of Rayleigh distribution is 0.448 for SNR at $30 \mathrm{~dB}$. For Rician distribution, the distance between the transmitters and the receivers can be varied because of the line of sight propagation. As they are in parallel planes, the channel coefficients can be calculated, and the respective received data can be detected by using the ZF equalizer. From Figure 5 we observe that the error rate increases as the distance from the transmitter to the receiver increases. As the link distance $d_{11}$ increased from $1 \mathrm{~m}$ to $100 \mathrm{~m}$, it is observed that the geometry of the channel model is varying, thereby introducing larger spherical and absorption losses, calculated by (16) and (17) respectively. Note that as the distance $d_{11}$ varies, all other link distances, which are used to calculate various channel coefficients $\left(h_{11}, h_{12}, h_{21}\right.$ and $\left.h_{22}\right)$ also vary. The phase shifts of the signals propagating in longer paths are recalculated by (14). It is observed that the BER is proportional to the considered distance.

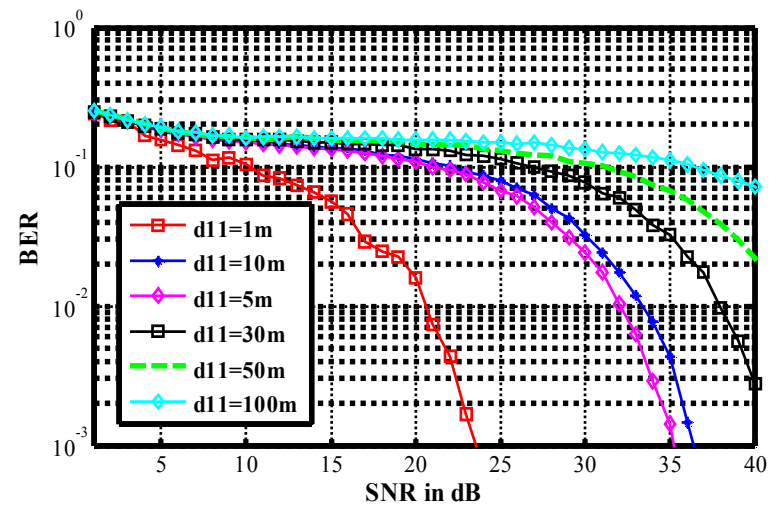

Fig. 5. SNR vs. BER of ZF equalization with different distances

The main intention of using the 4-QAM modulation scheme is its efficiency to use the bandwidth. Generally, whenever the requirement of linearity of a modulation scheme like QAM increases, the power efficiency of the current power amplifiers considerably reduces. So a simple 4-QAM modulation is used in this case of transmission. In Figure 6 we observe that the 4QAM is the best utilized modulation scheme. Though the higher modulation orders give higher data rates, they are very resilient to noise and interference. Therefore, there is a small deterioration in the performance of 4-QAM between 15 to $23 \mathrm{~dB}$ of SNR. As SNR decreases, errors will increase along with data retransmitting, thereby slowing throughput. By reverting to a lower order modulation scheme the link can be made more reliable with fewer data errors and retransmittions. So, the 4-QAM is used for better efficiency and reduced complexity.

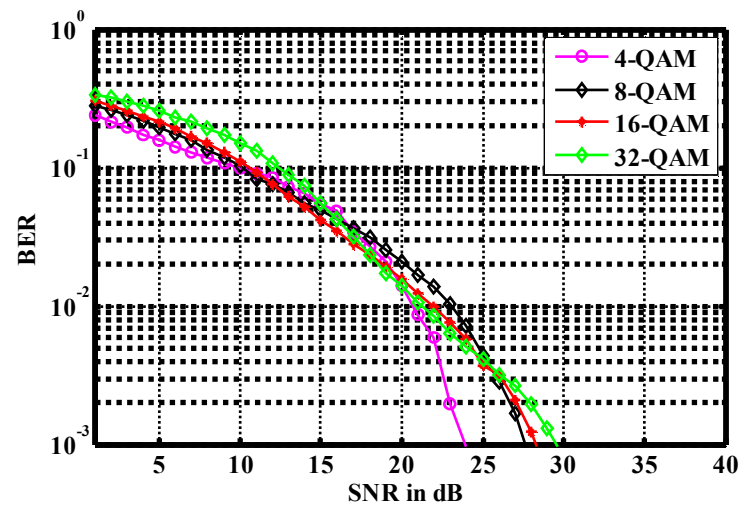

Fig. 6. SNR vs. BER for different states of QAM 


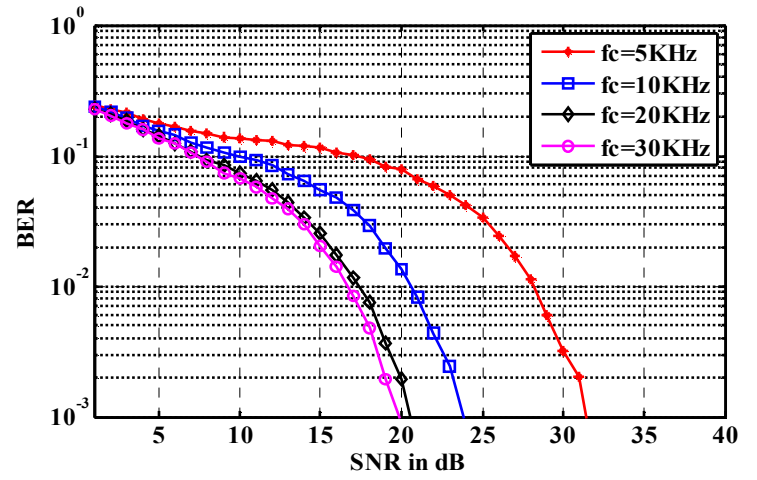

Fig. 7. SNR vs. BER for different carrier frequencies

Figure 7 gives the SNR to BER curve for various carrier frequencies. We observe that the number of errors keeps on decreasing as the carrier frequency value increases. Carrier frequency and the relative motion between the transmitter and the receiver depend upon the Doppler effect. By optimizing the choice of these basis functions, the Doppler shift is estimated. Doppler effect plays an important role in the efficient design of high data rate acoustic communication systems.

\section{CONCLUSIONS AND FUTURE WORK}

Simulation results prove that the LOS component of UWAC outperforms the NLOS component. All the scattering effects of the undersea channel characteristics are considered in this work. Moreover, in LOS propagation, the performance on varying distances is calculated. Results have been presented for various states of the QAM modulation scheme and also for different carrier frequencies. The shallow water acoustic channel is highly time-varying and does not assuredly follow a Rayleigh distribution. High data rates, high performance and optimum utilization of the bandwidth are the ultimate goals of the future generation UWAC. Many other equalization techniques, which further improve the performance of the underwater system, have to be implemented in this realistic channel, which considers absorption and spherical coefficients. In the future, we aim to develop robust long-range communication in the deep sea with higher data rates based on the proposed modem hardware. Real-time detection of undersea environments has to be studied further. Also, MIMO systems that handle more than two transmitters are to be investigated.

\section{ACKNOWLEDGMENT}

The authors are grateful to the administration of National Institute of Technology, Warangal.

\section{REFERENCES}

[1] G. Qiao, S. Liu, Z. Sun, F. Zhou, "Full-duplex, multi-user and parameter reconfigurable underwater acoustic communication modem", OceansSan Diego, San Diego, USA, September 23-26, 2013

[2] Y. Emami, R. Javidan, "An energy-efficient data transmission scheme in underwater wireless sensor networks", Engineering, Technology \& Applied Science Research, Vol. 6, No. 2, pp. 931-936, 2016

[3] A. Khasawneh, M. S. B. A. Latiff, O. Kaiwartya, H. Chizari, "A reliable energy-efficient pressure-based routing protocol for the underwater wireless sensor network", Wireless Networks, Vol. 24, No. 6, pp. 20612075,2018
[4] O. Kaiwartya, A. H. Abdullah, Y. Cao, J. Lloret, S. Kumar, R. R. Shah, M. Prasad, S. Prakash, "Virtualization in wireless sensor networks: Fault tolerant embedding for the internet of things", IEEE Internet of Things Journal, Vol. 5, No. 2, pp. 571-580, 2018

[5] M. A. B. Yusof, S. Kabir, "An overview of sonar and electromagnetic waves for underwater communication”, IETE Technical Review, Vol. 29, No. 4, pp. 307-317, 2012

[6] D. Wen, W. Cai, Y. Pan, "Design of underwater optical communication system", OCEANS 2016-Shanghai, Shanghai, China, April 10-13, 2016

[7] A. Ranjan, A. Ranjan, "Underwater wireless communication network", Advance in Electronic and Electric Engineering, Vol. 3, No. 1, pp. 4146, 2013

[8] A. Khasawneh, M. S. B. A. Latiff, O. Kaiwartya, H. Chizari, "Next forwarding node selection in underwater wireless sensor networks (UWSNs): Techniques and challenges", Information, Vol. 8, No. 1, p. 3, 2016

[9] A. Elsanousi, S. Ozturk, "Performance analysis of OFDM and OFDMMIMO systems under fading channels", Engineering, Technology \& Applied Science Research, Vol. 8, No. 4, pp. 3249-3254, 2018

[10] C. Krishnaswamy, N. Sireesha, T. Chinnasamy, V. Gowthaman, S. S. Narayanan, T. Sudhakar, M. Atmanand, "Underwater communication implementation with OFDM", Indian Journal of Geo-Marine Sciences, Vol. 44, No. 2, pp. 259-266, 2015

[11] W. B. Yang, T. C. Yang, "Characterization and modeling of underwater acoustic communications channels for frequency-shift-keying signals", Oceans 2006, Boston, USA, September 18-21, 2006

[12] O. Kaiwartya, A. H. Abdullah, Y. Cao, R. S. Raw, S. Kumar, D. K. Lobiyal, I. F. Isnin, X. Liu, R. R. Shah, "T-MQM: Testbed-based multimetric quality measurement of sensor deployment for precision agriculture-A case study", IEEE Sensors Journal, Vol. 16, No. 23, pp. 8649-8664, 2016

[13] C. Raj, R. Sukumaran, "Stochastic network calculus for rician fading in underwater wireless networks", Applied Mathematics \& Information Sciences, Vol. 10, No. 4, pp. 1465-1474, 2016

[14] L. Zhang, M. Li, G. Li, "Symbol estimation for MIMO underwater acoustic communication based on a multiplicative noise model", IEEE International Conference on Communication Problem-Solving, Beijing, China, December 5-7, 2014

[15] D. B. Kilfoyle, J. C. Preisig, A. B. Baggeroer, "Spatial modulation experiments in the underwater acoustic channel", IEEE Journal of Oceanic Engineering, Vol. 30, No. 2, pp. 406-415, 2005

[16] B. Li, J. Huang, S. Zhou, K. Ball, M. Stojanovic, L. Freitag, P. Willett, "MIMO-OFDM for high-rate underwater acoustic communications", IEEE Journal of Oceanic Engineering, Vol. 34, No. 4, pp. 634-644, 2009

[17] L. Zhang, M. Li, G. Li, R. Wang, "Symbol estimation algorithm for MIMO underwater acoustic communication system based on a multiplicative noise model", Mathematical Problems in Engineering, Vol. 2015, Article ID 719025, 2015

[18] T. Karp, S. Trautmann, N. J. Fliege, "Zero-forcing frequency-domain equalization for generalized DMT transceivers with insufficient guard interval", EURASIP Journal on Applied Signal Processing, Vol. 10, pp. 1446-1459, 2004

[19] B. Pranitha, H. L. Minh, N. Aslam, L. Anjaneyulu, S. Vangal, "BER performance investigation of MIMO underwater acoustic communications", 11th International Symposium on Communication Systems, Networks \& Digital Signal Processing, Budapest, Hungary, July 18-20, 2018

[20] K. Sankhe, S. Chaudhari, G. R. Murthy, "Distributed spatial modulation with dynamic frequency allocation", Physical Communication, Vol. 23, pp. 65-75, 2017

[21] K. M. Humadi, A. I. Sulyman, A. Alsanie, "Spatial modulation concept for massive multiuser MIMO systems", International Journal of Antennas and Propagation, Vol. 2014, Article ID 563273, 2014

[22] G. Qiao, Z. Babar, L. Ma, S. Liu, J. Wu, "MIMO-OFDM underwater acoustic communication systems: a review", Physical Communication, Vol. 23, pp. 56-64, 2017 
[23] Manjiti, R. Kaushik, "MIMO-OFDM and IDMA scheme in underwater communication", International Journal of Innovative Technology and Research, Vol. 4, No. 3, pp. 2971-2976, 2016

[24] J. Poncela, M. C. Aguayo, P. Otero, "Wireless underwater communications", Wireless Personal Communications, Vol. 64, No. 3, pp. 547-560, 2012

[25] M. R. C. Raj, R. Sukumaran, "Modeling Rayleigh fading channel in underwater wireless communication networks using stochastic network calculus", Journal of the Indian Mathematical Society, Vol. 84, No. 1-2, pp. 29, 2017

[26] B. Pranitha, L. Anjaneyulu, "Review of research trends in underwater communications-A technical survey", International Conference on Communication and Signal Processing, Melmaruvathur, India, April 6-8, 2016

[27] H. Esmaiel, D. Jiang, "Multicarrier communication for underwater acoustic channel", International Journal of Communications, Network and System Sciences, Vol. 6, pp. 361-376, 2013

[28] M. Naderi, M. Patzold, A. G. Zajic, “A geometry-based channel model for shallow underwater acoustic channels under the rough surface and bottom scattering conditions", IEEE Fifth International Conference on Communications and Electronics, Danang, Vietnam, July 30-August 1, 2014

[29] D. V. Ha, N. V. Duc, M. Patzold, "SINR analysis of OFDM systems using a geometry-based underwater acoustic channel model", 26th Annual International Symposium on Personal, Indoor, and Mobile Radio Communications, Hong Kong, China, August 30-September 2, 2015 